\title{
Modelagem Computacional de Efeitos Sensoriais
}

\author{
Renato Rodrigues $^{1}$, Felipe Henriques ${ }^{1}$, José Ricardo $^{2}$ \\ Diego Brandão ${ }^{1}$, Joel dos Santos ${ }^{1}$ \\ ${ }^{1}$ PPCIC - CEFET/RJ - Rio de Janeiro - RJ - Brasil \\ ${ }^{2}$ IFRJ - Rio de Janeiro - RJ - Brasil
}

\begin{abstract}
Resumo. A inclusão de efeitos sensoriais em aplicações multimídia interativas apresenta o desafio de se controlar diversos aspectos da reprodução desses efeitos em conjunto com sua representação no ambiente virtual e a interação do usuário. Utilizar técnicas de dinâmica de fluidos computacionais para simular os efeitos sensoriais e usar estes dados para controlar os atuadores pode facilitar a autoria de tais aplicações e promover uma melhor experiência aos usuários. Este trabalho apresenta algumas das diferentes abordagens para a simulação de fluidos, bem como uma implementação para ser utilizada no contexto de aplicações interativas e demonstra diferentes visualizações de dados da simulação para estudar seu comportamento.
\end{abstract}

\section{Introdução}

Aplicações multimídia que possibilitam a apresentação de efeitos sensoriais - e.g., aroma, temperatura, etc. - sincronizados com outros objetos de mídia tradicionais são denominadas aplicações mulsemídia (do inglês mulsemedia - Multiple Sensorial Media) [Ghinea et al. 2014]. Tais aplicações têm o potencial de aprimorar a qualidade de experiência do usuário durante o consumo de conteúdo multimídia [Timmerer et al. 2012]. Alguns trabalhos investigam o uso de efeitos sensoriais em aplicações interativas apresentando melhorias na qualidade de experiência dos usuários [Kahol et al. 2006, Apostolopoulos et al. 2012] e na retenção de informação pelos usuários [Tortell et al. 2007].

As dificuldades associadas na inclusão de estímulos sensoriais em aplicações interativas são decorrentes da necessidade de controlar os dispositivos que reproduzem os efeitos sensoriais - i.e., os atuadores - juntamente com a simulação destes efeitos no ambiente virtual e sua mudança de estado de acordo com a interação do usuário. Suponha que em uma aplicação, uma determinada região do mundo virtual possui um aroma associado. O usuário caminha pelo mundo virtual até esta região. Paralelamente, o atuador presente no ambiente real deve emitir esse efeito seguindo sua dispersão no ambiente virtual - i.e. a intensidade deverá variar de acordo com a posição do usuário no ambiente virtual.

Neste contexto, utilizar técnicas de dinâmica de fluidos computacional para a simulação dos efeitos sensoriais e utilizar os dados desta simulação para controlar os dispositivos atuadores pode auxiliar no desenvolvimento de tais aplicações e proporcionar uma melhor experiência aos usuários. Este trabalho tem como objetivo investigar as diferentes abordagens de simulação de fluidos, apresentar uma implementação de uma destas técnicas para ser utilizada em aplicações mulsemídia e apresentar diferentes visualizações 
de dados das características desta simulação com o objetivo de identificar o comportamento da simulação e corrigir seu parâmetros, caso necessário.

O restante deste artigo está estruturado da seguinte forma: a Seção 2 descreve as diferentes abordagens para simulação de fluidos, a Seção 3 apresenta os trabalhos relacionados, a Seção 4 apresenta a metodologia utilizada para a geração da visualização de dados, a Seção 5 apresenta os resultados das visualizações, e por fim, a Seção 6 apresenta as conclusões.

\section{Simulação de fluidos}

Em aplicações mulsemídia os efeitos sensoriais de maior interesse são aqueles que provém estímulos táteis e olfativos, visto que os efeitos audiovisuais já são contemplados por aplicações multimídia tradicionais. A dificuldade em reproduzir estes efeitos sensoriais é manter aspectos como posição e intensidade do efeito sensorial constantemente atualizados de acordo com a interação do usuário com o mundo virtual. Alguns destes efeitos sensoriais comportam-se como fluidos - e.g., vento, aroma e spray. Desta forma, é possível simular estes fluidos computacionalmente e utilizar as informações da simulação tanto para a renderização dos efeitos sensoriais nas cenas quanto para controlar os atuadores de acordo com o comportamento dos fluidos durante a execução da aplicação.

As equações da Navier-Stokes modelam matematicamente o movimento da maioria dos fluidos não compressíveis que ocorrem na natureza [Wesseling 2009]. Uma simplificação destas equações pode ser definida da seguinte forma:

$$
\begin{gathered}
\nabla \cdot u=0 \\
\frac{\partial u}{\partial t}=-(u \cdot \nabla) u-\frac{1}{\rho} \nabla p+v \nabla^{2} u+F
\end{gathered}
$$

A Equação 1 é denominada equação de continuidade e define que a massa do sistema deve ser conservada. A Equação 2 é denominada equação de movimento e define a aceleração de um fluido dadas as forças atuantes. Duas abordagens podem ser utilizadas para a discretização do espaço ou domínio da simulação: métodos baseados em malhas ou métodos baseados em partículas, também conhecido como métodos Meshfree [da Silva Junior 2010].

Nos métodos baseados em malha, toda a superfície é representada por um conjunto de células, onde cada célula contém um conjunto de vetores das forças atuantes sobre o fluido formando um campo vetorial. A Figura 1(a) apresenta uma representação bidimensional de uma malha, onde $p$ representa a pressão e $u$ representa a velocidade do fluido. As malhas podem ser representadas por uma descrição espacial Euleriana ou descrição material Lagrangeana [Liu and Liu 2003]. A Tabela 1 apresenta uma comparação entre os dois métodos.

Nos métodos baseados em partículas, os fluidos são representados por um conjunto de partículas. O Smoothed Particles Hydrodynamics (SPH) é um método baseado em partículas, desenvolvido originalmente para aplicações de astrofísica [Lucy 1977, 


\begin{tabular}{lll}
\hline & Método Lagrangiano & Método Euleriano \\
\hline Malha & Fixa no material & Fixa no espaço \\
Rastreio & Movimento de qualquer ponto no material & Massa, movimento e fluxo de energia entre células na malha \\
Histórico temporal & Fácil de obter & Difícil de obter \\
Geometrias irregulares & Fáceis de modelar & Difíceis de modelar \\
Grandes deformações & Difíceis & Fáceis \\
\hline
\end{tabular}

Tabela 1. Comparação entre os métodos Lagrangiano e Euleriano [Liu and Liu 2003]

Gingold and Monaghan 1977] mas também utilizado para simulações computacionais [Müller et al. 2003], onde cada partícula armazena as informações das forças atuantes sobre ela e estas forças exercem influência sobre as outras partículas de acordo com uma função de vizinhança. A Figura 1(b) ilustra a disposição das partículas no espaço e a representação da função de vizinhança.

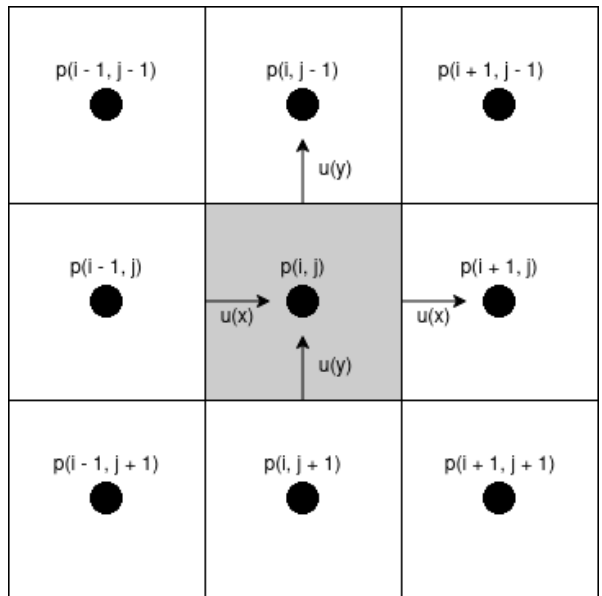

(a) Representação bidimensional da malha

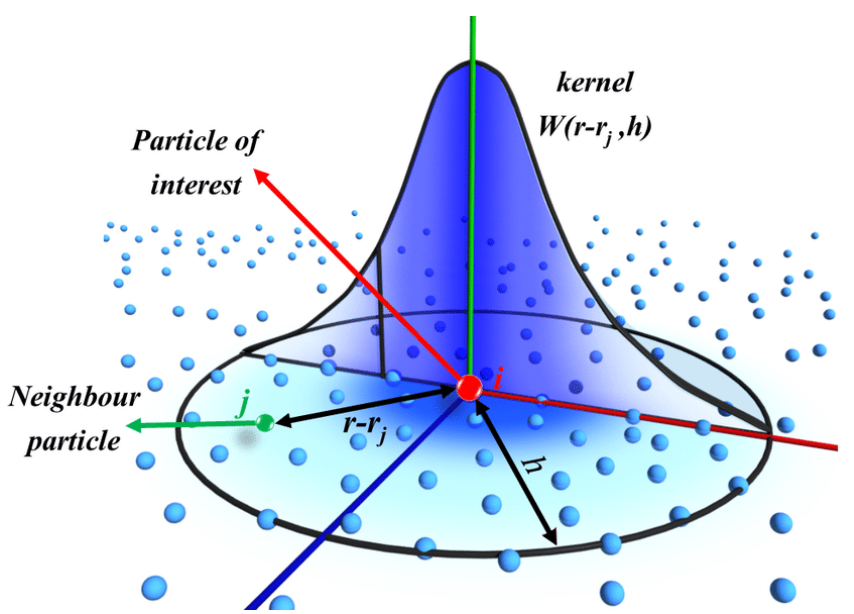

(b) Representação das partículas e função de vizinhança [Giorgi 2018]

Figura 1. Abordagens de discretização do espaço ou domínio da simulação

Ambos os métodos apresentam vantagens e desvantagens em diferentes cenários dependendo de suas aplicações. No geral, os métodos de malhas são mais complexos computacionalmente pois a malha deve compreender todo o ambiente da simulação enquanto os métodos de partículas são mais eficientes pois a representação é contida apenas no fluido. No entanto, os métodos de malhas produzem simulações mais fieis a realidade do que os métodos de partículas. Além disso, a precisão e estabilidade do método de partículas é dependente da densidade de partículas por área. No contexto de mulsemídia, há um maior interesse em produzir simulações interativas em tempo real que apresentam uma precisão boa o suficiente para permitir o ajuste dos atuadores - dispositivos que por si só não apresentam grande precisão - do que uma que simule perfeitamente a realidade. Desta forma, o método SPH será utilizado neste trabalho para simulações de fluidos.

\section{Trabalhos relacionados}

Nesta seção são discutidos os trabalhos relacionados com a proposta apresentada neste artigo. Os trabalhos estão organizados naqueles que abordam $(i)$ simulação computacional de efeitos sensoriais e (ii) simulação de fluidos para aplicações interativas. Em geral, 
os trabalhos relacionados que tratam da simulação computacional de efeitos sensoriais abordam a dispersão de aromas em um ambiente virtual.

[Ishida et al. 2008, Matsukura et al. 2009] propõem a utilização de uma simulação de dinâmica de fluidos computacional (CFD, do inglês Computational Fluid Dynamics), em conjunto com um dispositivo emissor de aromas para calcular o fluxo de ar em um ambiente e controlar a dispersão do aroma. Nos artigos são apresentadas duas demonstrações, uma apresentando o aroma em sincronia com um vídeo e outra gerando a difusão do aroma de acordo com um fluxo de ar variável. O software CFD2000 foi utilizado para realizar os cálculos da simulação dos fluidos. Os dados são armazenados e utilizados posteriormente nas simulações.

[Matsukura and Ishida 2009] descrevem algumas considerações para obter uma experiência mais realista identificada a partir de algumas discrepâncias apresentadas nos resultados dos experimentos realizados nos artigos apresentados anteriormente. Um dos fatores considerados no artigo indica que a percepção de aromas é diferente para cada indivíduo, portanto a intensidade do efeito sensorial deve ser adaptada de acordo a sensibilidade olfativa. Outro fator importante é considerar a presença de um corpo humano na simulação uma vez que a convecção causada pela temperatura do corpo induz mudanças significativas na concentração do aroma.

[Nakamoto et al. 2020] desenvolveram uma aplicação de realidade virtual onde o usuário busca pela fonte de um odor. $\mathrm{O}$ ambiente virtual consiste de um prédio de dois andares com oito salas, sendo quatro salas em cada andar, e em uma das salas está localizado a fonte do odor. Uma simulação de CFD utilizando o software ANSYS18.1 no supercomputador TSUBAME 3.0 foi executada para calcular a difusão do odor. Os dados da simulação são armazenados para serem utilizados posteriormente durante a execução da aplicação. O usuário, equipado de óculos de realidade virtual e um dispositivo emissor de aroma, caminha pelo ambiente para encontrar a fonte de odor. A intensidade do aroma emitido pelo dispositivo varia de acordo com os dados gerados pela CFD em relação a posição do usuário no ambiente e a duração da aplicação.

Nos trabalhos relacionados apresentados, as simulações computacionais de efeitos sensoriais são realizadas utilizando métodos de malhas, com a computação feita antes da execução da aplicação e armazenada para ser consultada posteriormente. Esta abordagem apresenta uma limitação ao impedir que possíveis mudanças que ocorram no ambiente virtual durante a execução da aplicação afetem a simulação dos efeitos sensoriais. Assim, seria desejado que a simulação ocorresse em tempo real. Nos parágrafos a seguir são apresentadas abordagens de simulação de fluidos interativas que podem ser executadas em tempo real utilizando o método SPH.

[Müller et al. 2003] apresentam um método baseado em partículas para simulação interativa de fluidos e renderização. O modelo físico é baseado no SPH e são definidas funções de vizinhança especiais para melhorar a estabilidade e velocidade de execução. No entanto, o método apresentado é mais adequado para fluidos não compressíveis, e.g., líquidos como água. Em se tratando da simulação de efeitos sensoriais, temos efeitos que se comportam como gases, e.g., aroma, névoa e vento.

A simulação de gases utilizando o método SPH é computacionalmente complexa pois é necessário adicionar partículas de ar do ambiente que exercerão suas forças so- 
bre as partículas do gás de interesse, aumentando muito o número de partículas na simulação. Neste contexto, [Ren et al. 2016] apresentam uma abordagem utilizando forças de compensação que eliminam a necessidade das partículas de ambiente na simulação conforme ilustra a Figura 2. Neste trabalho será utilizada esta abordagem para a simulação de um gás.

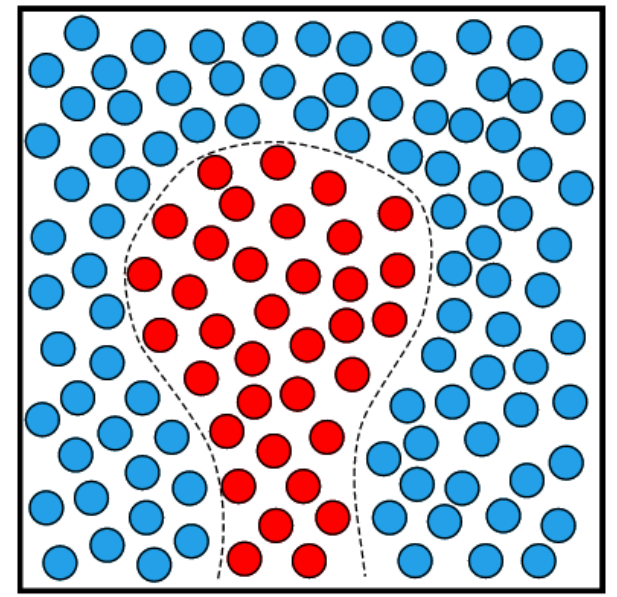

(a)

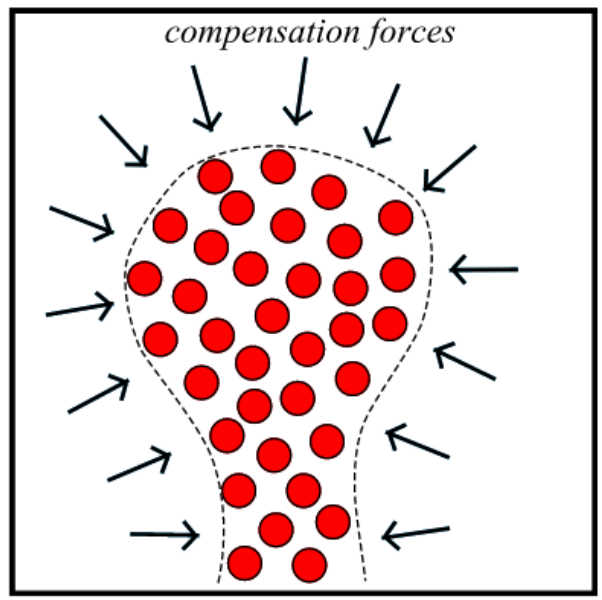

(b)

Figura 2. (a) Abordagem padrão com a simulação preenchida por partículas de ar do ambiente (em azul). (b) Abordagem utilizando forças de compensação [Ren et al. 2016]

\section{Metodologia}

Afim de produzir visualizações de dados para auxiliar no desenvolvimento da simulação de fluidos proposta, foram analisadas as possíveis variáveis contidas nas partículas para definir quais podem produzir visualizações interessantes. As variáveis selecionadas para produzir visualizações foram: temperatura, densidade, velocidade, aceleração e posição. As variáveis possuem tipos diferentes; temperatura e densidade são número reais, velocidade e aceleração são vetores tridimensionais, e a posição é um ponto no espaço tridimensional. Como estas variáveis possuem tipos diferentes elas devem ser tratadas de diferentes maneiras para serem representadas na simulação. Números reais são mapeados diretamente na escala de cor, os vetores são computados dos tamanhos produzindo um número real para mapeamento, e a posição é mapeada diretamente na escala RGB (onde o eixo $x, y$ e $z$ representam o vermelho, verde e azul, respectivamente).

Para colorir as partículas para a visualização é utilizado o sistema de cores HSV (matiz, saturação e valor, do inglês hue, saturation e value). Para os valores da matiz são computados os limites inferiores e superiores dos valores das variáveis obtidos durante a simulação e mapeados entre 0 e 360 . A saturação utilizada é de $70 \%$ e o valor de $50 \%$. Esta configuração produz uma variação de cores que vai do azul até o vermelho, representando os valores mais baixos com as cores mais frias (mais próximas ao azul(, e os valores mais altos com as cores mais quentes (mais próximas ao vermelho).

\section{Resultados}

A Figura 3 ilustra as diferentes visualizações com as variáveis selecionadas. 


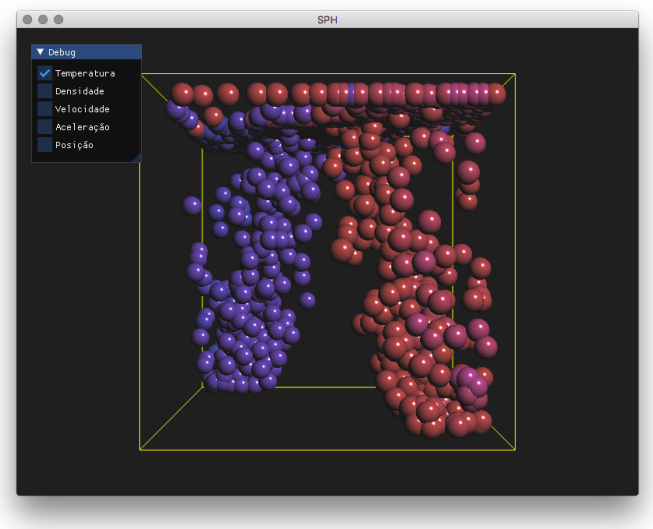

(a)

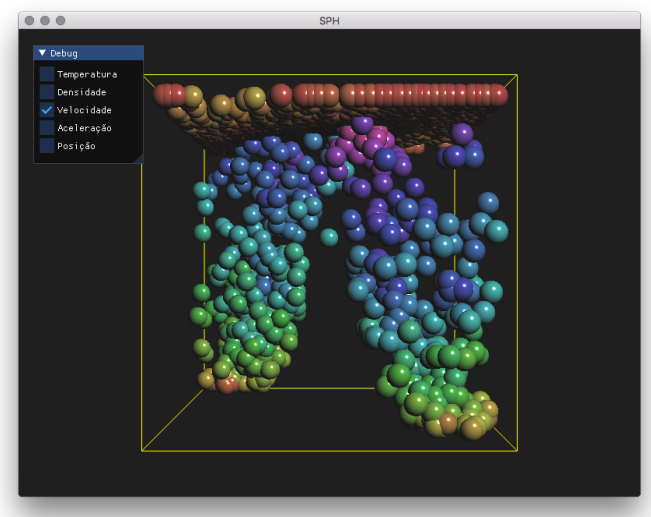

(c)

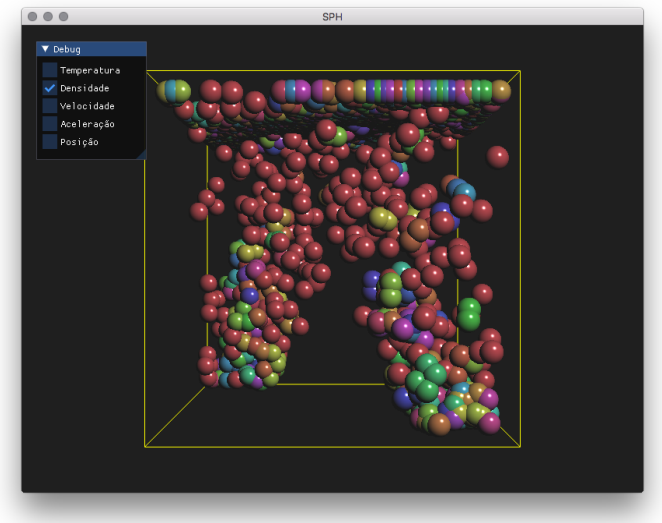

(b)

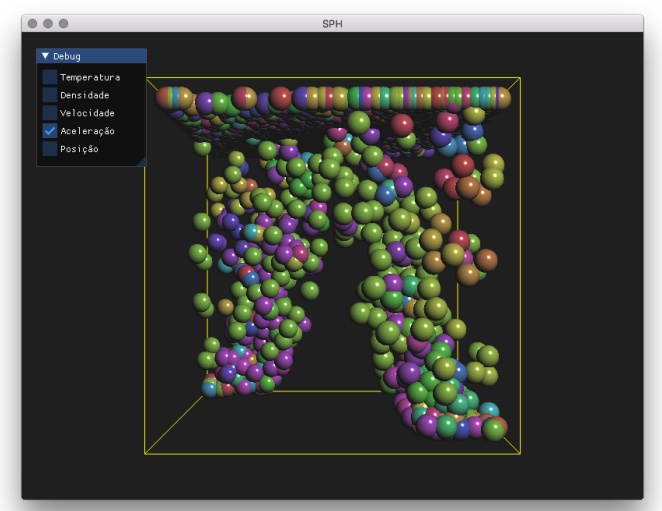

(d)

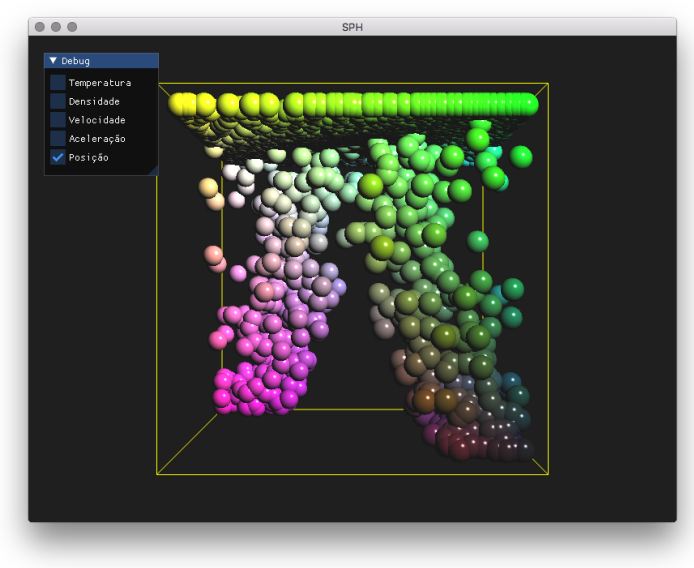

(e)

Figura 3. (a) Temperatura. (b) Densidade. (c) Velocidade. (d) Aceleração. (e) Posição.

A Figura 3(a) representa a visualização da variação da temperatura entre as partículas. Com esta visualização foi possível observar que a implementação da troca de temperatura entre as partículas está funcionando de maneira adequada. Na simulação, 
uma extremidade do ambiente emite partículas mais frias (azul) e na outra partículas mais quentes (vermelho). Conforme as partículas se encontram há a troca de calor e dado o avanço da simulação as partículas assumem uma temperatura intermediária com uma cor próxima ao roxo.

A Figura 3(b) representa a visualização da densidade. Esta visualização nos permite confirmar um comportamento esperado da simulação, que é a alta variação da densidade, dada sua constante atualização.

A Figura 3(c) representa a visualização da velocidade. Na simulação, as partículas começam com uma velocidade intermediária, perdem um pouco de velocidade ao ganhar altitude e atingem sua maior velocidade ao chegar no topo do ambiente da simulação. Esta visualização permitiu observar que há algum parâmetro na simulação que deve ser ajustado, o que justifica as partículas se acumularem no topo pois estão com uma velocidade muito alta. As partículas quentes deveriam se concentrar no topo e as partículas mais frias deveriam ficar abaixo das partículas quentes.

A Figura 3(d) representa a visualização da aceleração. Assim como a visualização da densidade, esta visualização permite confirmar o funcionamento esperado da simulação, i.e., a interação entre as partículas promove uma alta variação da aceleração.

Por fim, a Figura 3(e) representa a visualização da posição. Esta visualização permite apenas localizar mais facilmente a dispersão das partículas no ambiente. Diferente das demais, não possui nenhuma informação significativa sobre os parâmetros da simulação.

\section{Conclusão}

Neste trabalho, apresentamos uma proposta de visualização de dados oriundos da modelagem computacional de efeitos sensoriais em aplicações multimídia. Conforme demonstrado, as visualizações apresentadas permitem identificar possíveis anomalias que ocorrem na simulação. Principalmente na implementação da troca de temperatura entre as partículas, onde foi possível identificar se a propriedade estava funcionando da maneira adequada. Além disso, foi possível identificar também um possível problema nos parâmetros utilizados na simulação que está causando o acumulo das partículas no topo do ambiente da simulação. Uma melhoria que pode ser feita na visualização é utilizar a representação de uma seta apontando na direção das variáveis que representam vetores tridimensionais.

\section{Referências}

Apostolopoulos, J. G., Chou, P. A., Culbertson, B., Kalker, T., Trott, M. D., and Wee, S. (2012). The road to immersive communication. Proceedings of the IEEE, 100(4):974990 .

da Silva Junior, J. R. (2010). Simulação computacional em tempo real de fluidos utilizando o método sph em ambiente heterogêneo cpu/gpu. Master's thesis, Universidade Federal Fluminense, UFF, Niterói, Brasil.

Ghinea, G., Timmerer, C., Lin, W., and Gulliver, S. R. (2014). Mulsemedia : State of the Art, Perspectives, and Challenges. ACM Transactions on Multimedia Computing, Communications, and Applications, 11(1s):1-23. 
Gingold, R. A. and Monaghan, J. J. (1977). Smoothed particle hydrodynamics: theory and application to non-spherical stars. Monthly notices of the royal astronomical society, 181(3):375-389.

Giorgi, G. (2018). Nonlinear hydrodynamic modelling of wave energy converters under controlled conditions. PhD thesis, National University of Ireland Maynooth.

Ishida, H., Matsukura, H., Yoshida, H., and Nakamoto, T. (2008). Application of computational fluid dynamics simulation to olfactory display. In Proc. ICAT, pages 285-288.

Kahol, K., Tripathi, P., Mcdaniel, T., Bratton, L., and Panchanathan, S. (2006). Modeling context in haptic perception, rendering, and visualization. ACM Transactions on Multimedia Computing, Communications, and Applications (TOMM), 2(3):219-240.

Liu, G.-R. and Liu, M. B. (2003). Smoothed particle hydrodynamics: a meshfree particle method. World scientific.

Lucy, L. B. (1977). A numerical approach to the testing of the fission hypothesis. The astronomical journal, 82:1013-1024.

Matsukura, H. and Ishida, H. (2009). Olfactory display: fluid dynamic considerations for realistic odor presentation. In Proceedings of the 15th Joint virtual reality Eurographics conference on Virtual Environments, pages 61-64.

Matsukura, H., Yoshida, H., Ishida, H., and Nakamoto, T. (2009). Interactive odor playback based on fluid dynamics simulation. In 2009 IEEE Virtual Reality Conference, pages 255-256. IEEE.

Müller, M., Charypar, D., and Gross, M. H. (2003). Particle-based fluid simulation for interactive applications. In Symposium on Computer animation, pages 154-159.

Nakamoto, T., Hirasawa, T., and Hanyu, Y. (2020). Virtual environment with smell using wearable olfactory display and computational fluid dynamics simulation. In 2020 IEEE Conference on Virtual Reality and 3D User Interfaces (VR), pages 713-720. IEEE.

Ren, B., Yan, X., Yang, T., Li, C.-f., Lin, M. C., and Hu, S.-m. (2016). Fast sph simulation for gaseous fluids. The Visual Computer, 32(4):523-534.

Timmerer, C., Waltl, M., Rainer, B., and Hellwagner, H. (2012). Assessing the quality of sensory experience for multimedia presentations. Signal Processing: Image Communication, 27(8):909-916.

Tortell, R., Luigi, D., Dozois, A., Bouchard, S., Morie, J. F., and Ilan, D. (2007). The effects of scent and game play experience on memory of a virtual environment. Virtual Reality, 11(1):61-68.

Wesseling, P. (2009). Principles of computational fluid dynamics, volume 29. Springer Science \& Business Media. 